\title{
Fructose-bisphosphate aldolase $A$ is a key regulator of hypoxic adaptation in colorectal cancer cells and involved in treatment resistance and poor prognosis
}

\author{
KENJI KAWAI $^{1}$, MAMORU UEMURA ${ }^{1,2}$, KOJI MUNAKATA ${ }^{1,3}$, HIDEKAZU TAKAHASHI $^{1}$, \\ NAOTSUGU HARAGUCHI ${ }^{1}$, JUNICHI NISHIMURA ${ }^{1}$, TAISHI HATA ${ }^{1}$, CHU MATSUDA $^{1}$, \\ MASAKAZU IKENAGA ${ }^{4}$, KOHEI MURATA ${ }^{5}$, TSUNEKAZU MIZUSHIMA ${ }^{1,6}$, \\ HIROFUMI YAMAMOTO $^{1,7}$, YUICHIRO DOKI ${ }^{1}$ and MASAKI MORI ${ }^{1}$
}

\begin{abstract}
${ }^{1}$ Department of Gastroenterological Surgery, Graduate School of Medicine, Osaka University, Suita, Osaka 565-0871;
${ }^{2}$ Department of Surgery, National Hospital Organization Osaka National Hospital, Chuo-ku, Osaka, Osaka 540-0006, Japan;

${ }^{3}$ Division of Gastroenterology, Department of Internal Medicine, University of Michigan, 3100A Taubman Center,

Ann Arbor, MI 48109-5368, USA; ${ }^{4}$ Department of Surgery, Higashi-osaka City General Hospital, Higashiosaka,

Osaka 578-8588; ${ }^{5}$ Department of Surgery, Suita Municipal Hospital, Suita, Osaka 564-0082; Departments of ${ }^{6}$ Therapeutics

for Inflammatory Bowel Diseases, and ${ }^{7}$ Molecular Pathology, Division of Health Sciences, Graduate School of Medicine,

Osaka University, Osaka 565-0871, Japan
\end{abstract}

Received October 21, 2016; Accepted November 23, 2016

DOI: $10.3892 /$ ijo.2016.3814

\begin{abstract}
Hypoxia is an essential feature of cancer malignancy, but there are no methods for the routine detection of hypoxiainducible prognostic factors and potential therapeutic targets. We reported previously that the hypoxic tumor cells of metastatic liver tissue from patients with colorectal cancer (CRC) could be used as an 'in vivo' hypoxia culture model. Several potential hypoxia-inducible genes were identified using this model. Among them, one glycolytic enzyme was of special interest. There is currently increasing attention on glycolytic enzymes as potential therapeutic targets due to their association with cancer-specific metabolism. To better understand the molecular mechanisms of cancer malignancy, we investigated
\end{abstract}

Correspondence to: Dr Mamoru Uemura, Department of Surgery, National Hospital Organization Osaka National Hospital, 2-1-14, Houenzaka, Chuo-ku, Osaka 540-0006, Japan

E-mail: uemura7777@hotmail.com

Abbreviations: ADM, adrenomedullin; ALDOA, fructose-bisphosphate aldolase A; CA9, carbonic anhydrase IX; CRC, colorectal cancer; DFS, disease-free survival; EFNA1, ephrin-A1; EMT, epithelial-mesenchymal transition; HIF1A, hypoxia-inducible factor 1-alpha; OS, overall survival; PCR, polymerase chain reaction; PI, propidium iodide; PLOD2, procollagen-lysine, 2-oxoglutarate 5-dioxygenase 2; RIPA, radio immunoprecipitation assay; SCGB2A1, secretoglobin, family $2 \mathrm{~A}$, member $1 ; 5$-FU, 5 -fluorouracil

Key words: fructose-bisphosphate aldolase A, chemoresistance, radioresistance, colorectal cancer, hypoxia the expression of fructose-bisphosphate aldolase A (ALDOA) and its relationship with cancer metabolism. We found that ALDOA was induced by hypoxia in CRC-derived cell lines, and univariate and multivariate analyses of microarray data from the resected CRC samples of 222 patients revealed that ALDOA was an independent prognostic factor for CRC. We also analyzed the malignant potential of ALDOA in vitro using overexpression and knockdown assays. We found that ALDOA was negatively related to chemosensitivity and radiosensitivity and positively associated with proliferation, sphere formation and invasion in both normoxia and hypoxia. These associations were due to the roles of ALDOA in regulating glycolysis, the epithelial-mesenchymal transition and the cell cycle. These findings demonstrate that ALDOA is a hypoxiainducible prognostic factor that is closely related to CRC malignancy, and also provide new insights into the importance of ALDOA and glycolysis in cancer and suggest new targets for anticancer therapies.

\section{Introduction}

Colorectal cancer (CRC) is one of the most frequent cancers in humans (1). Despite recent advances and new treatments, including combination therapy with anticancer and molecular targeted drugs $(2,3)$, CRC remains a major cause of cancer morbidity and mortality (4). Thus, there is still a need to identify new therapeutic targets in CRC.

In the tumor microenvironment, cellular hyperplasia and relative blood flow insufficiency often lead to tumor hypoxia. To survive in this harsh environment, cancer cells develop adaptive responses to hypoxia that are mediated by the activation of hypoxia-inducible genes. In this context, hypoxia is a major feature of cancer, and in hypoxic conditions, cancer cells 
can develop into malignant cells that survive in the low-oxygen environment. Previous reports have suggested associations between hypoxia and solid tumors, including brain tumors (5), breast (6), liver (7), lung 8), ovarian (9), prostate (10) and CRC (11).

Microarray techniques allow researchers to detect hypoxia-induced genes. Most hypoxia-related experiments are based on in vitro conditions. However, the problem with hypoxic models is that there are apparent differences between the experimental hypoxic models and actual in vivo hypoxic environments. In experimental hypoxic conditions, there are many unpreventable biases, such as the degree of hypoxia, the duration of the hypoxic conditions, $\mathrm{pH}$, growth factors and nutrients. Moreover, it is almost impossible to replicate the complex cellular interactions that occur in vivo in an in vitro environment.

We reported previously that liver metastasis of CRC is like 'in vivo' hypoxia culture box, and that it is useful for identifying hypoxia-inducible potential prognostic factors and therapeutic targets (12). We used this model to identify the following novel hypoxia-inducible genes: Jumonji domain containing 1A (JMJD1A) (12), adrenomedullin (ADM) (13), ephrin-A1 (EFNA1) (14), procollagen-lysine, 2-oxoglutarate 5-dioxygenase 2 (PLOD2) (15) and secretoglobin, family 2A, member 1 (SCGB2A1) (16).

In the present study, we investigated the involvement of fructose-bisphosphate aldolase A (ALDOA) in CRC. ALDOA is one of the glycolytic enzymes that catalyzes the reversible conversion of fructose-1,6-bisphosphate to glyceraldehyde3-phosphate and dihydroxyacetone phosphate (17). Notably, previous studies have reported that ALDOA is a downstream target of hypoxia-inducible factor 1-alpha (HIF1A) $(18,19)$, and several studies have reported a relationship between ALDOA and cancers such as osteosarcoma $(20,21)$, lung squamous cell carcinoma (22) and hepatocellular carcinoma (23).

The aim of the present study was to investigate the prognostic value and the biological significance of ALDOA in CRC. We determined the expression of ALDOA in clinical samples and performed functional analyses of ALDOA using overexpression and knockdown assays. Furthermore, our data suggest possible mechanisms that underlie the correlation of ALDOA with cancer malignancy.

\section{Materials and methods}

Cell lines and culture conditions. Six human CRC cell lines were used in this study. HCT116 and LoVo cells were purchased from the Japanese Collection of Research Bioresources Cell Bank (JCRB; Tokyo, Japan), and HT29 and SW480 cells were purchased from the American Type Culture Collection (ATCC; Manassas, VA, USA). DLD-1 cells were purchased from the Cell Resource Center for the Biomedical Research Institute of Development, Aging, and Cancer at Tohoku University. The KM12SM cells were the kind gift of Professor T. Minamoto (Kanazawa University, Ishikawa, Japan). Authenticity of all cells was confirmed. Cells were cultured in Dulbecco's modified Eagle's medium containing 10\% fetal bovine serum (FBS), $100 \mathrm{U} / \mathrm{ml}$ penicillin and $100 \mu \mathrm{g} / \mathrm{ml}$ streptomycin in a $37^{\circ} \mathrm{C}$ incubator supplied with $5 \% \mathrm{CO}_{2}$. Hypoxic culture conditions $\left(1 \% \mathrm{O}_{2}\right)$ were achieved in a multi-gas incubator (Panasonic
MCO-5MUV; Panasonic, Osaka, Japan) using a continuous infusion of a gas mixture $\left(95 \% \mathrm{~N}_{2}, 5 \% \mathrm{CO}_{2}\right)$.

Ethics statement regarding the use of clinical samples. The use of clinical samples was approved by the Human Ethics Review Committee of the Graduate School of Medicine, Osaka University. Written informed consent was obtained from all patients included in the study.

Clinical tissue samples. For western blot analysis, 6 surgical CRC samples from patients with no preoperative chemotherapy or irradiation were randomly selected from samples collected at Osaka University Hospital in 2016. For immunohistochemical staining, 14 surgical samples of colorectal liver metastases from patients with no preoperative chemotherapy or irradiation were collected from 2012 to 2015 at the Osaka University Hospital.

Clinical sample collection and microarray analysis. Microarray analysis was performed as previously described $(12,16,24)$. A total of 222 clinical samples were collected from consecutive patients who underwent curative (R0) resection for CRC from 2003 to 2006 at the Osaka University Hospital and its nine associated hospitals. The mean follow-up time was $40.2 \pm 26.4$ months for patients with disease-free survival (DFS) and 52.7 \pm 18.5 months for all surviving patients (overall survival, OS). Table I shows the clinicopathological features of the patients, including gender, tumor location, depth of invasion, lymph node metastasis, histological grade, Dukes' stage and vessel invasion. None of the patients whose samples were analyzed in this study received preoperative chemotherapy or irradiation. After surgery, patients with Dukes' stage $\mathrm{C}$ tumors were generally treated with 5-fluorouracil (5-FU)-based chemotherapy.

Quantitative PCR. mRNA expression was determined by realtime polymerase chain reaction (PCR). Total RNA was isolated using an RNeasy Maxi kit (Qiagen, Valencia, CA, USA), and reverse transcription was performed using the reverse transcription system (Promega, Madison, WI, USA) according to the manufacturer's instructions. The real-time PCR analysis was performed using the LightCycler 2.0 instrument system (Roche Diagnostics, Mannheim, Germany), based on Thunderbird SYBR qPCR Mix (Toyobo Co., Ltd., Osaka, Japan). The $\beta$-actin housekeeping gene was used as an internal control. The following primers were used in the present study: ALDOA: forward 5'-CTGCCAGTATGTGACCGAGA-3' and reverse 5'-ACAGGAAGGTGATCCCAGTG-3'; HIF1A: forward 5'-GAAAGCGCAAGTCCTCAAAG-3' and reverse 5'-TGGGTAGGAGATGGAGATGC-3'; vimentin: forward 5'-CCCTCACCTGTGAAGTGGAT-3' and reverse 5'-TCCA GCAGCTTCCTGTAGGT-3'; and $\beta$-actin: forward 5'-GATG AGATTGGCATGGCTTT-3' and reverse 5'-CACCTTCACC GTTCCAGTTT-3'. The analysis was performed using the standard curve method. For data analysis, raw counts were normalized to the housekeeping gene average for the same time-point and condition.

Western blotting. Proteins were extracted from the cells and clinical samples in radio-immunoprecipitation assay (RIPA) 
Table I. The relationship between ALDOA expression and clinicopathological factors in patients with colorectal cancer ( $\mathrm{n}=222)$.

\begin{tabular}{|c|c|c|c|c|}
\hline & \multirow[b]{2}{*}{ Total $(n=222)$} & \multicolumn{3}{|c|}{ ALDOA expression } \\
\hline & & Low $(\mathrm{n}=111)$ & High $(n=111)$ & P-value \\
\hline Age (years); mean \pm SD & $66.2 \pm 10.0$ & $65.3 \pm 9.6$ & $67.1 \pm 10.2$ & 0.24 \\
\hline \multicolumn{5}{|l|}{ Gender } \\
\hline Male/female & $133 / 89$ & $65 / 46$ & $68 / 43$ & 0.78 \\
\hline \multicolumn{5}{|l|}{ Tumor location } \\
\hline Colon/rectum & $141 / 81$ & $68 / 43$ & $73 / 38$ & 0.58 \\
\hline \multicolumn{5}{|l|}{ Depth of invasion } \\
\hline$\leq \mathrm{mp} / \geq \mathrm{sS}$ & $27 / 195$ & $12 / 99$ & $15 / 96$ & 0.68 \\
\hline \multicolumn{5}{|l|}{ Lymph node metastasis } \\
\hline Present/absent & $109 / 113$ & $45 / 66$ & $64 / 47$ & $0.016^{\mathrm{a}}$ \\
\hline \multicolumn{5}{|l|}{ Histological grade } \\
\hline Well differentiated/other & $53 / 169$ & $27 / 84$ & $26 / 85$ & 1 \\
\hline \multicolumn{5}{|l|}{ Dukes' stage } \\
\hline $\mathrm{AB} / \mathrm{CD}$ & $110 / 112$ & $67 / 44$ & $43 / 68$ & $0.002^{\mathrm{a}}$ \\
\hline \multicolumn{5}{|l|}{ Vessel invasion } \\
\hline Present/absent & $184 / 38$ & $89 / 22$ & $95 / 16$ & 0.37 \\
\hline
\end{tabular}

mp, muscularis propria; ss, subserosa. ${ }^{\mathrm{a}} \mathrm{P}<0.05$.

buffer (Thermo Fisher Scientific, Waltham, MA, USA). Clinical samples were homogenized in RIPA buffer using an ultrasonic disruptor (Ultrasonic Disruptor UD-201; Tomy Seiko, Co., Ltd., Tokyo, Japan). Proteins were separated by electrophoresis on SDS-PAGE Tris-HCl gels (Bio-Rad Laboratories, Hercules, CA, USA). The separated proteins were transferred onto polyvinylidene difluoride membranes and blocked with 5\% skim milk in Tris-buffered saline with Tween-20 (TBS-T) at room temperature for $1 \mathrm{~h}$. The blots were incubated with primary antibodies overnight and then with HRP-linked anti-rabbit or anti-mouse IgG (GE Healthcare Biosciences, Piscataway, NJ, USA) at a dilution of 1:100,000 for $1 \mathrm{~h}$ at room temperature. The antigen-antibody complex was detected with the ECL Prime Western blotting detection kit (GE Healthcare Biosciences).

Antibodies for western blot analysis. Western blot analyses were carried out using the following antibodies: anti-ALDOA (\#3188; Cell Signaling Technology, Danvers, MA, USA); anti-E-cadherin (sc-7870; Santa Cruz Biotechnology, Inc., Santa Cruz, CA, USA); anti-vimentin (\#5741; Cell Signaling Technology) and anti-ACTB (A2066; Sigma-Aldrich, St. Louis, MO, USA).

Immunohistochemical staining. Paraffin-embedded tissue sections $(4-\mu \mathrm{m})$ were deparaffinized. After antigen retrieval treatment, immunohistochemical staining was performed using the Vectastain ABC Peroxidase kit (Vector Laboratories, Burlingame, CA, USA). The tissue sections were then incubated overnight at $4^{\circ} \mathrm{C}$ with antibodies at the following dilutions: anti-ALDOA, 1:100 (ab169544; Abcam, Cambridge,
MA, USA) and anti-CA9, 1:100 (\#5649; Cell Signaling Technology).

Expression vector transfection. Cell lines were transfected with an ALDOA expression vector (pCMV6-XL5 vector, SC108418; Origene Technologies, Inc., Rockville, MD, USA) using the FuGENE ${ }^{\circledR} 6$ transfection reagent (Promega) according to the manufacturer's recommended protocol. The same method was used to transfect cells with an empty vector as negative controls.

Small interfering RNA transfection. Cell lines were transfected with $20 \mathrm{nM}$ siRNA targeting ALDOA expression (Silencer Select siRNA, siRNA ID s71, cat. no. 4390824; Life Technologies, Carlsbad, CA, USA: 5'-AGUCCCUCUUCGU CUCUAATT-3' and 5'-UUAGAGACGAAGAGGGACUAG-3') using Lipofectamine ${ }^{\circledR}$ RNAiMAX transfection reagent (Invitrogen, Carlsbad, CA, USA) according to the manufacturer's recommended protocol. The same method was used to transfect cells with negative control siRNA (Silencer Select negative control\#1 siRNA, cat. no. 4390843; Life Technologies).

Proliferation assay. The proliferation assay was performed as previously described (16). ALDOA overexpression cells and knockdown cells plus controls for each cell line were seeded and cultured in a $37^{\circ} \mathrm{C}$ incubator supplied with $5 \% \mathrm{CO}_{2}$. After $24,48,72$ and $96 \mathrm{~h}$ of incubation, cell viability was measured using the Cell Counting kit-8 (CCK-8; Dojindo Laboratories, Kumamoto, Japan) according to the manufacturer's recommended protocol. CCK-8 solution was added to each well and incubated for $2 \mathrm{~h}$. 
Chemosensitivity assay. The chemosensitivity assay was performed as previously described (16). Cell viability was measured after $48 \mathrm{~h}$ of incubation in drug-containing medium at $\mathrm{IC}_{50}$ concentrations.

Radiosensitivity assay. The radiosensitivity assay was performed as previously described (16). Cells were exposed to 8-Gy single-dose irradiation using a Gammacell 40 Exactor ${ }^{137} \mathrm{Cs}$ radiation source (Nordion, Inc., Ottawa, ON, Canada) and cell viability was measured after $48 \mathrm{~h}$ of incubation in normal medium.

Sphere formation assay. The ability of cells to form spheres was evaluated in Dulbecco's modified Eagle's medium/ Nutrient Mixture F-12 (DMEM/F12) supplemented with $20 \mathrm{ng} / \mathrm{ml}$ epidermal growth factor (Invitrogen), $20 \mathrm{ng} / \mathrm{ml}$ human platelet growth factor (Sigma-Aldrich) and 1\% antibiotic-antimycotic solution (Invitrogen). Single cells were plated at a concentration of 100 cells/well in each well of a 96-well ultralow attachment plate (Corning Life Sciences, Acton, MA, USA) and cultured in a $37^{\circ} \mathrm{C}$ incubator supplied with $5 \% \mathrm{CO}_{2}$. The number of spheres $\geq 100 \mu \mathrm{m}$ in each well was counted to evaluate the sphere-forming ability.

Invasion assay. The invasion assay was performed using Corning ${ }^{\circledR}$ BioCoat ${ }^{\mathrm{TM}}$ Matrige ${ }^{\circledR}$ Invasion Chambers (Corning Life Sciences). Briefly, 25,000 cells were seeded in triplicate onto the Matrigel-coated membrane. After $48 \mathrm{~h}$, cells that had invaded the undersurface of the membrane were fixed and stained with Diff-Quik (Sysmex Internal Reagents, Co., Ltd., Kobe, Japan). Three microscopic fields were randomly selected for cell counting.

Lactate production assay. Lactate production was measured using the Lactate Colorimetric Assay kit II (BioVision, Inc., Milpitas, CA, USA). Cells were seeded in 12-well plates (Corning Life Sciences) at densities of 100,000 cells (DLD-1) and 150,000 cells (HT29 and KM12SM) per well. After 24 h, the cells were transfected with siRNA targeting ALDOA or with an ALDOA expression vector, and then $48 \mathrm{~h}$ after transfection, the subconfluent cells were washed three times with phosphate-buffered saline (PBS). Krebs-Ringer bicarbonate buffer containing $10 \mathrm{mM}$ glucose was added, and the amount of lactate was assessed 30 min later in aliquots of media from each well. The absorbance at $450 \mathrm{~nm}$ was then measured using the Bio-Rad Model 680 XR microplate reader (Bio-Rad Laboratories).

Apoptosis assay. Apoptosis was measured by flow cytometry using the Annexin V-FITC apoptosis kit (BioVision) according to the manufacturer's recommended protocol.

The samples were analyzed using BD FACSAria IIu $^{\mathrm{TM}}$ instrument (BD Biosciences, San Jose, CA, USA). Both early apoptotic (Annexin V-FITC-positive, PI-negative) and necrotic/late apoptotic (Annexin V-FITC-positive, PI-positive) cells were quantified as apoptotic cells.

Analysis of reactive oxygen species (ROS). ROS production was measured using the CellROX ${ }^{\circledR}$ Deep Red reagent (Invitrogen) according to the manufacturer's recommended protocol.
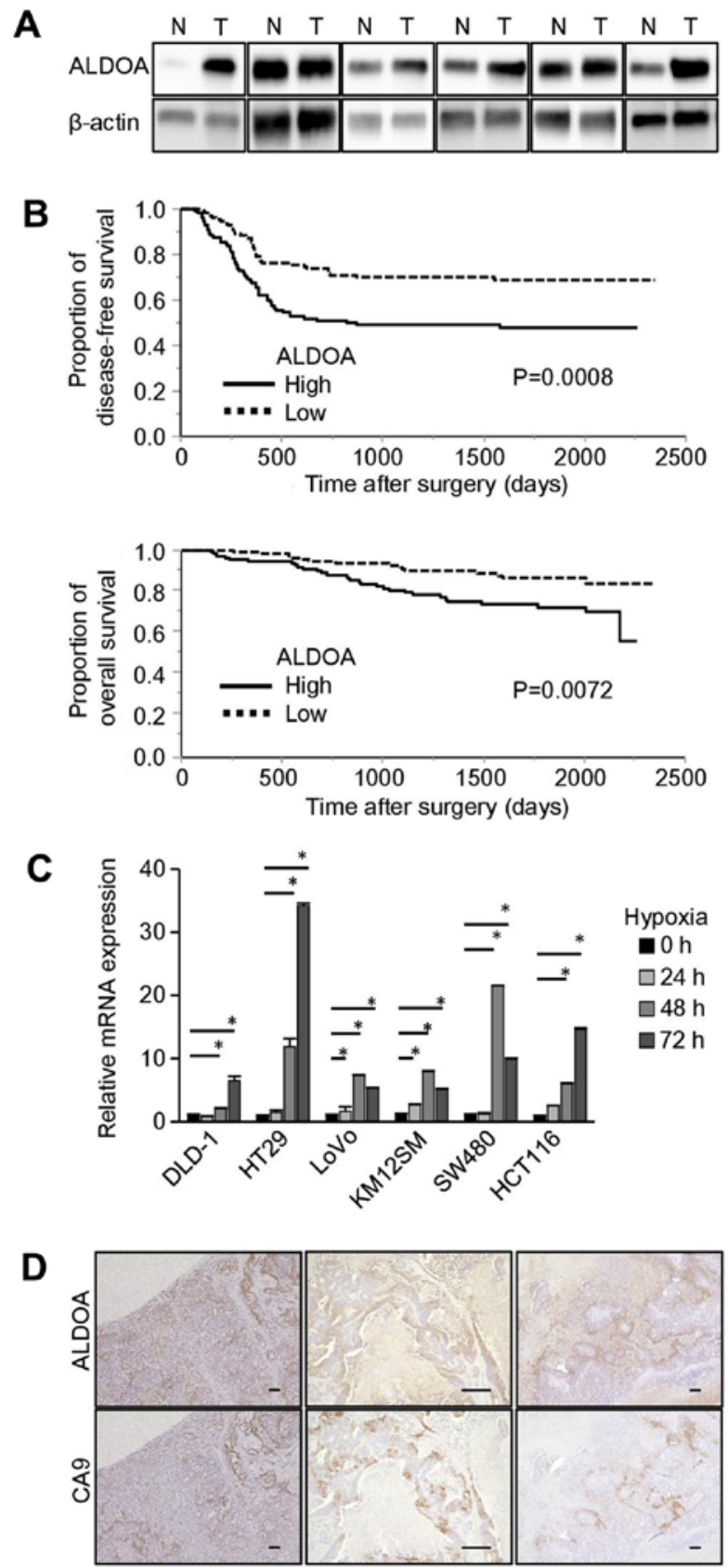

Figure 1. ALDOA expression is associated with cancer. (A) Western blot analysis of ALDOA protein expression in normal mucosa $(\mathrm{N})$ and tumor tissue (T) in 6 surgical samples. (B) Kaplan-Meier survival curves of the ALDOA high- and low-expression groups (log-rank test). (C) ALDOA mRNA expression in colorectal cancer (CRC) cell lines under hypoxia. Bars represent the means \pm SD of three independent experiments. ${ }^{*} \mathrm{P}<0.05$, Student's t-test. (D) Representative images of immunohistochemical staining of colorectal cancer liver metastases. Scale bar, $100 \mu \mathrm{m}$. CA9, carbonic anhydrase IX.

Fluorescence was measured using the BD FACSAria IIu instrument (BD Biosciences).

Cell cycle analysis. Cell cycle phase distribution was analyzed by flow cytometry using PI staining. Briefly, cells were fixed in $70 \%$ ethanol on ice for $30 \mathrm{~min}$. Fixed cells were incubated in a solution containing $0.1 \mathrm{mg} / \mathrm{ml}$ RNase $\mathrm{A}$ at $37^{\circ} \mathrm{C}$ for $20 \mathrm{~min}$ and then in a solution containing $25 \mu \mathrm{g} / \mathrm{ml} \mathrm{PI}$ on ice for $2 \mathrm{~min}$. Fluorescence was measured immediately using 
Table II. Univariate and multivariate analysis of clinicopathological factors associated with disease-free survival in patients with colorectal cancer $(n=222)$.

\begin{tabular}{|c|c|c|c|c|}
\hline & \multirow{2}{*}{$\begin{array}{l}\text { Univariate } \\
\text { analysis } \\
\text { P-value }\end{array}$} & \multicolumn{3}{|c|}{ Multivariate analysis } \\
\hline & & Relative risk & $95 \% \mathrm{CI}$ & P-value \\
\hline \multicolumn{5}{|l|}{ Tumor location } \\
\hline Colon/rectum & $0.036^{\mathrm{a}}$ & 0.592 & $0.288-0.908$ & $0.0167^{\mathrm{a}}$ \\
\hline \multicolumn{5}{|l|}{ Depth of invasion } \\
\hline$\leq \mathrm{mp} / \geq \mathrm{sS}$ & $0.0058^{\mathrm{a}}$ & 0.313 & $0.093-0.781$ & $0.01^{\mathrm{a}}$ \\
\hline \multicolumn{5}{|l|}{ Lymph node metastasis } \\
\hline Present/absent & $<0.0001^{\mathrm{a}}$ & 1.324 & $0.852-2.100$ & 0.2149 \\
\hline \multicolumn{5}{|l|}{ Histological grade } \\
\hline Well differentiated/others & $0.0193^{\mathrm{a}}$ & 0.704 & $0.379-1.212$ & 0.2147 \\
\hline \multicolumn{5}{|l|}{ Dukes' stage } \\
\hline $\mathrm{AB} / \mathrm{CD}$ & $<0.0001^{\mathrm{a}}$ & & & \\
\hline \multicolumn{5}{|l|}{ Vessel invasion } \\
\hline Present/absent & $0.0002^{\mathrm{a}}$ & 3.311 & $0.090-0.763$ & $0.0088^{\mathrm{a}}$ \\
\hline \multicolumn{5}{|l|}{ ALDOA } \\
\hline High/low & 0.0008 & 2.01 & $1.310-3.127$ & $0.0013^{\mathrm{a}}$ \\
\hline
\end{tabular}

mp, muscularis propria; ss, subserosa; CI, confidence interval. ${ }^{\mathrm{P}}<0.05$.

the BD FACSAria IIu instrument. Flow cytometry data were analyzed using FlowJo software (Tree Star, Inc., Ashland, OR, USA).

Statistical analysis. Data management and statistical analysis were performed using the JMP software (version 11.0; SAS Institute, Inc., Cary, NC USA). Tests for associations were performed using the Student's t-tests and Chi-squared tests. The cumulative patient OS and DFS were compared using the Kaplan-Meier technique, and log-rank tests and Cox's model were used to assess the risk ratio for multivariate analysis. A $\mathrm{P}<0.05$ was considered statistically significant.

\section{Results}

Relationship between ALDOA expression and clinicopathological features. Western blot analysis indicated that in 5 out of 6 randomly selected CRC surgical samples, ALDOA expression was higher in tumor tissue than in normal colon mucosa (Fig. 1A). Then, the $222 \mathrm{CRC}$ patients were divided into two groups according to the ALDOA mRNA expression level in the tumor samples. The relationships between ALDOA mRNA expression and clinicopathological features were analyzed (Table I). The high ALDOA expression group included more patients with lymph node metastasis than the low expression group $(\mathrm{P}=0.016)$, as for other clinicopathological factors, there were no significant differences.

ALDOA mRNA expression level is significantly associated with DFS and OS. DFS and OS were significantly longer in the low expression group than in the high expression group (DFS: $\mathrm{P}=0.0008$, OS: $\mathrm{P}=0.0072$; Fig. 1B). Univariate and multivariate analysis showed that ALDOA mRNA expression was a significant prognostic factor for DFS $(\mathrm{P}=0.0013$; Table II $)$ and for $\mathrm{OS}(\mathrm{P}=0.0124$; Table III).

ALDOA expression is upregulated under hypoxic conditions. In all of the CRC cell lines we examined, ALDOA mRNA expression was significantly increased after 48 to $72 \mathrm{~h}$ of culture in hypoxic conditions compared with the levels in cells cultured in normoxic conditions (Fig. 1C). Immunohistochemical staining of CRC liver metastases showed that the regions stained by the anti-ALDOA antibody were almost the same as the regions stained by an antibody to carbonic anhydrase IX (CA9), which is an endogenous hypoxia marker (Fig. 1D).

ALDOA expression correlates with proliferative potential in CRC cells. PCR and western blot analysis demonstrated high transfer efficiency of the ALDOA expression vector and of the siRNA targeting ALDOA (data not shown). In the CRC-derived cell lines DLD-1 HT29 and KM12SM, upregulation of ALDOA expression induced significant cell proliferation compared to controls at $72 \mathrm{~h}(\mathrm{P}<0.05$; Fig. 2A), and downregulation of ALDOA expression reduced cell proliferation compared to controls at $72 \mathrm{~h}(\mathrm{P}<0.05$; Fig. 2B).

ALDOA confers chemoresistance and radioresistance. Upregulation of ALDOA significantly reduced chemosensitivity to 5-FU in DLD-1 and HT29 cells and to oxaliplatin in all three cell lines compared to controls ( $\mathrm{P}<0.05$; Fig. $2 \mathrm{C}$ ). 

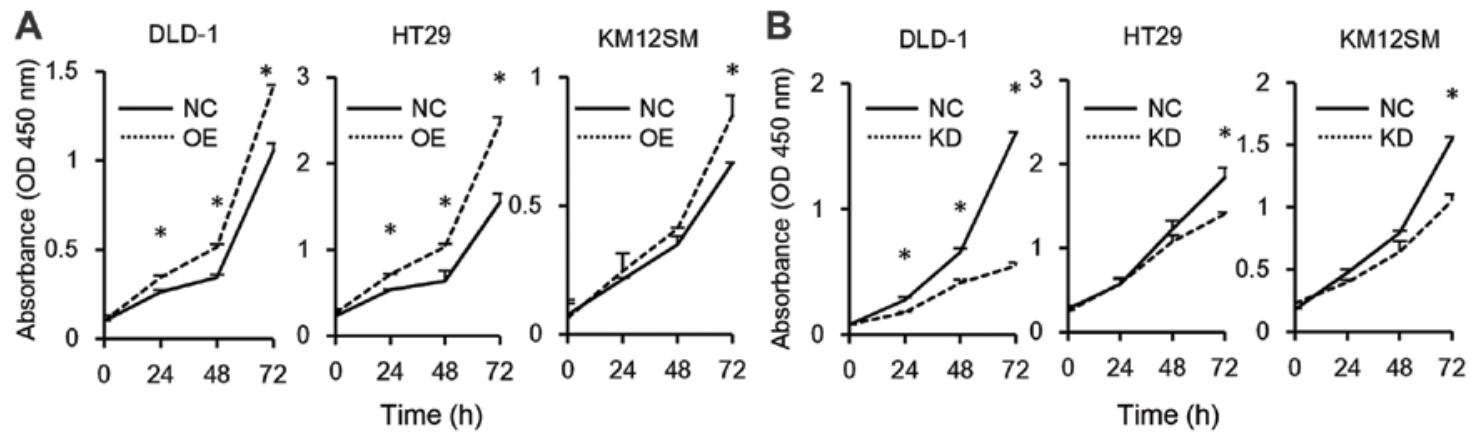

C

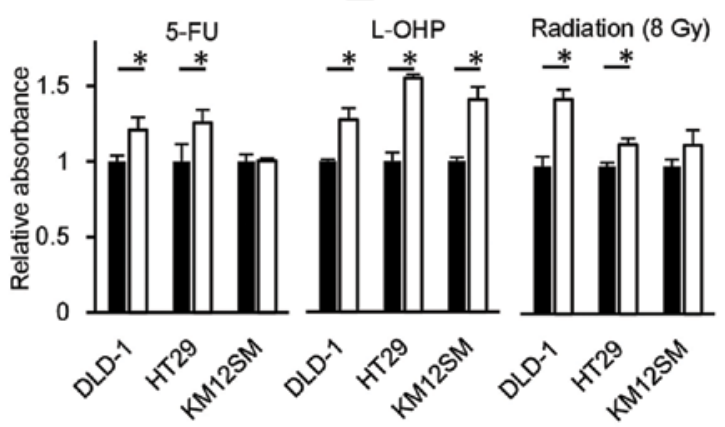

E

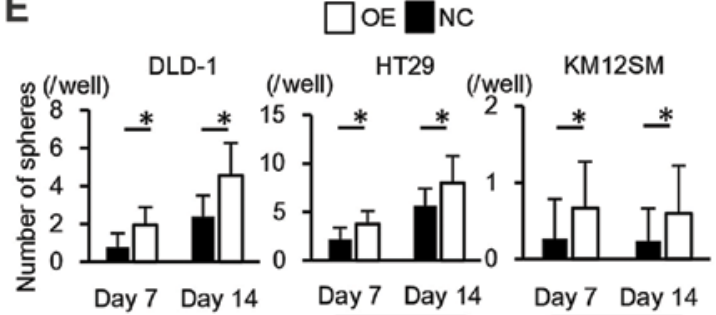

D

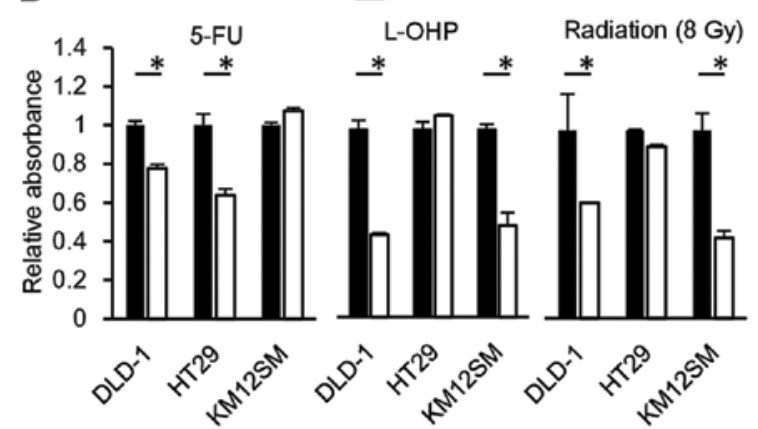

$\mathbf{F}$

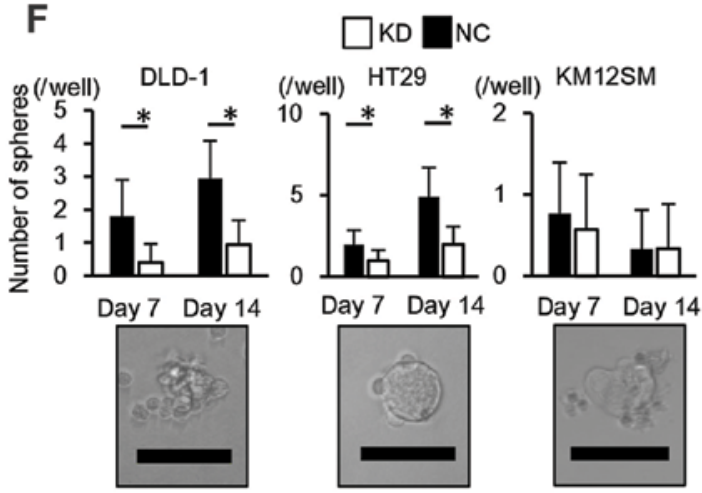

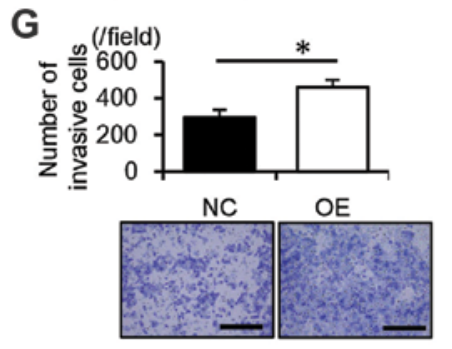
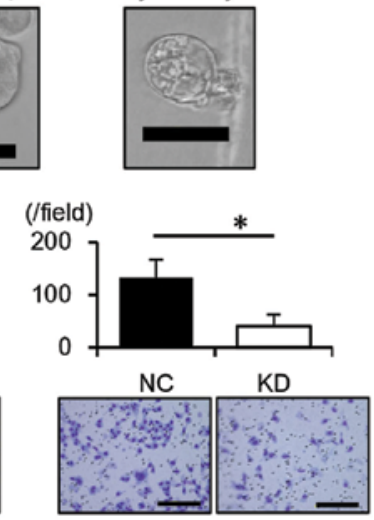

Figure 2. ALDOA is associated with the malignant potential of cancer. (A) The proliferation of cells transfected with the ALDOA expression vector. (B) The proliferation of cells transfected with siRNA targeting ALDOA expression. (C) The chemosensitivity and radiosensitivity of cells transfected with the ALDOA expression vector. (D) The chemosensitivity and radiosensitivity of cells transfected with siRNA targeting ALDOA expression. (E) Sphere formation by cells transfected with the ALDOA expression vector. Lower panel, representative images of a sphere; scale bar, $100 \mu \mathrm{m}$. (F) Sphere formation by cells transfected with siRNA targeting ALDOA expression. Lower panel, representative images of a sphere; scale bar, $100 \mu \mathrm{m}$. (G) Invasion by ALDOA overexpression cells (left) and ALDOA knockdown cells (right). Lower panel, representative images of the undersurface of the membrane; scale bar, $200 \mu \mathrm{m}$. The results represent the means \pm SD of three independent experiments. 5-FU, 5-fluorouracil; KD, ALDOA knockdown; L-OHP, oxaliplatin; NC, negative control; OE, ALDOA overexpression. ${ }^{*} \mathrm{P}<0.05$, Student's t-test.

In contrast, ALDOA downregulation significantly induced chemosensitivity to 5-FU in DLD-1 and HT29 cells and to oxaliplatin in DLD-1 and KM12SM cells compared to controls $(\mathrm{P}<0.05$; Fig. 2D). Upregulation of ALDOA significantly reduced radiosensitivity in DLD-1 and HT29 cells and that downregulation significantly induced radiosensitivity in DLD-1 and KM12SM cells compared to controls $(\mathrm{P}<0.05$; Fig. 2C and D).
Correlation of ALDOA expression with sphere formation. DLD-1, HT29 and KM12SM cells that overexpressed ALDOA were more tumorigenic than controls $(\mathrm{P}<0.05$; Fig. 2E). In DLD-1 and HT29, ALDOA knockdown cells were less tumorigenic than controls $(\mathrm{P}<0.05$; Fig. $2 \mathrm{~F})$.

ALDOA induces cell invasion. DLD-1 cells that overexpressed ALDOA invaded the Matrigel-coated membrane significantly 
Table III. Univariate and multivariate analysis of clinicopathological factors of overall survival in patients with colorectal cancer $(n=222)$.

$$
\begin{gathered}
\begin{array}{c}
\text { Univariate } \\
\text { analysis }
\end{array} \\
\text { P-value }
\end{gathered}
$$

0.8715

$$
\leq \mathrm{mp} / \geq \mathrm{ss}
$$

Lymph node metastasis

$$
\text { Present/absent }
$$$$
<0.0001^{\mathrm{a}}
$$

Histological grade

Well differentiated/others

Dukes' stage

$$
\mathrm{AB} / \mathrm{CD}
$$

$$
<0.0001^{\mathrm{a}}
$$

Vessel invasion
Multivariate analysis

Relative risk $\quad 95 \% \mathrm{CI} \quad$ P-value

Present/absent

ALDOA

$$
0.0237^{\mathrm{a}}
$$

$0.0072^{\mathrm{a}}$
High/low

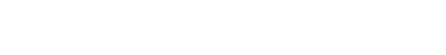

1.635

0.444-10.579

0.498

mp, muscularis propria; ss, subserosa; CI, confidence interval. ${ }^{\mathrm{P}} \mathrm{P}<0.05$. faster than control cells $(\mathrm{P}<0.05)$ and ALDOA knockdown cells invaded significantly more slowly than control $(\mathrm{P}<0.05$; Fig. 2G).

ALDOA expression is necessary for cancer cells to grow in hypoxic conditions. To examine the role of ALDOA in hypoxia, the proliferation and sphere formation assay were performed in hypoxic conditions. DLD-1 cells transfected with siRNA targeting ALDOA barely proliferated and formed very few spheres compared to controls $(\mathrm{P}<0.05$; Fig. $3 \mathrm{~A}$ and $\mathrm{B})$. Similarly, in the other cell lines, ALDOA knockdown cells formed hardly any spheres compared to controls $(\mathrm{P}<0.05$; Fig. 3B), collectively indicating that ALDOA is necessary for proliferation and tumor formation in hypoxic conditions. In addition, PCR analysis showed that HIF1A expression is upregulated by knockdown of ALDOA, probably because of a negative feedback loop (Fig. 3C).

ALDOA expression is proportional to the activity of glycolytic pathway. In DLD-1, HT29 and KM12SM cells, the knockdown of ALDOA resulted in a decrease in lactate production compared to controls, and, in contrast, its overexpression resulted in an increase in lactate production ( $\mathrm{P}<0.05$; Fig. 3D). This means that the expression of ALDOA and the activity of the glycolytic pathway were positively correlated.

ALDOA expression is associated with EMT progression. ALDOA knockdown assay was performed under hypoxia to assess the function of ALDOA in hypoxic conditions. To focus on the relationship between ALDOA and epithelial-mesen- chymal transition (EMT), we performed western blot analysis to assess the expression of EMT markers in ALDOA knockdown DLD-1 and HT29 cells cultured under normoxic and hypoxic conditions. We found that E-cadherin expression was reduced by hypoxia and induced by knockdown of ALDOA both in normoxia and hypoxia. As for mesenchymal markers, vimentin expression level was too low to detect on western blots (Fig. 4A). PCR revealed that vimentin mRNA expression was reduced by ALDOA knockdown in both normoxia and hypoxia $(\mathrm{P}<0.05$; Fig. 4B).

Knockdown of ALDOA stops cell cycle progression and enhances apoptosis due to rapid accumulation of ROS. The rate of apoptosis was higher in hypoxic DLD-1 and HT29 cells than in normoxic cells. It also showed that the rate of apoptosis was higher in ALDOA knockdown cells than in controls in both hypoxia and normoxia $(\mathrm{P}<0.05$; Fig. 5A). In addition, the ROS-positive cell rate of ALDOA knockdown cells was higher than controls both in hypoxia and in normoxia $(\mathrm{P}<0.05$; Fig. 5B). Cell cycle analysis showed that the G2/G1 ratio was higher in ALDOA knockdown cells than in controls in both hypoxia and normoxia ( $\mathrm{P}<0.05$; Fig. $5 \mathrm{C})$.

\section{Discussion}

There has been increasing interest in glycolytic enzymes as potential therapeutic targets because the cancer-specific metabolism depends more on the glycolytic pathway than on aerobic respiration, a phenomenon called the Warburg effect (25). The present report is the first to characterize the 
A

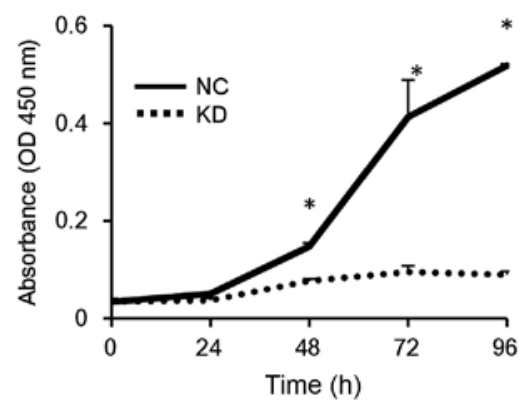

B

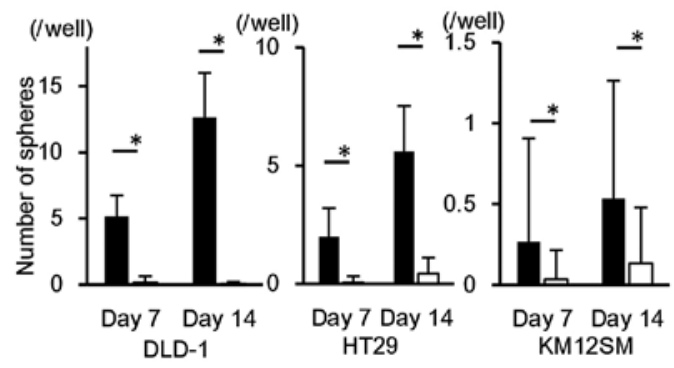

C

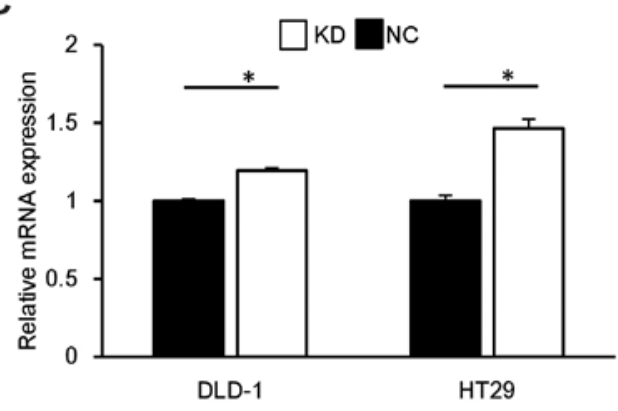

D

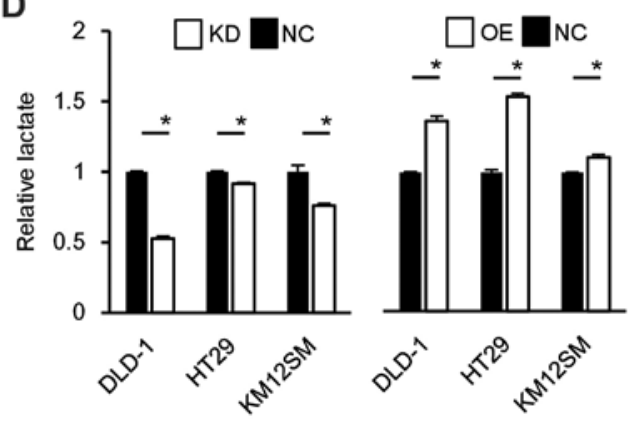

Figure 3. ALDOA is required for cancer cells to grow in hypoxic conditions, and the expression is proportional to the activity of glycolytic pathway. (A) Proliferation of DLD-1 cells transfected with siRNA targeting ALDOA expression performed under hypoxia. (B) Sphere formation by cells transfected with siRNA targeting ALDOA expression cultured under hypoxia. (C) HIF1A mRNA expression by cells transfected with siRNA targeting ALDOA expression. (D) Aliquots of the medium from cultures of ALDOA knockdown cells (left) and ALDOA overexpression cells (right) were removed at the indicated time points and tested for lactate. The results represent the means \pm SD of three independent experiments. HIF1A, hypoxia-inducible factor 1-alpha; KD, ALDOA knockdown; LDHA, lactate dehydrogenase A; NC, negative control; OE, ALDOA overexpression. ${ }^{*} \mathrm{P}<0.05$, Student's t-test.

importance of ALDOA in CRC and to investigate in detail the possible mechanisms underlying that relationship.

The most meaningful finding in the present study was that there is a close association between ALDOA expression and the clinicopathological features and the malignant potential
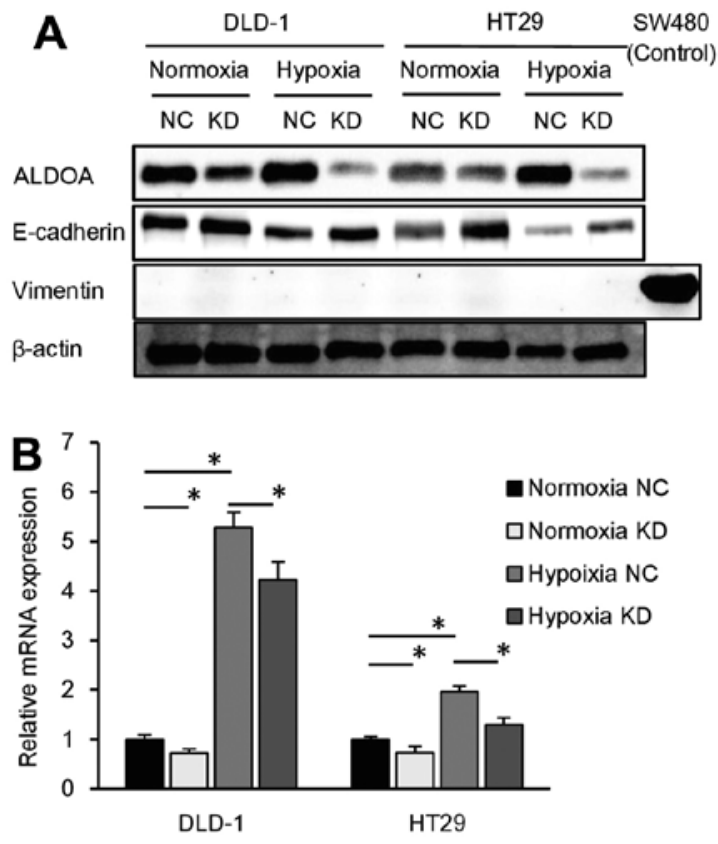

Figure 4. ALDOA expression is associated with the epithelial-mesenchymal transition (EMT). (A) Western blot analysis of the EMT markers E-cadherin and vimentin in ALDOA knockdown cells in normoxic and hypoxic conditions. SW480 was used as a positive control for vimentin. (B) Vimentin mRNA expression in ALDOA knockdown cells in normoxic and hypoxic conditions. The results represent the means $\pm \mathrm{SD}$ of three independent experiments. KD, ALDOA knockdown; NC, negative control. * $\mathrm{P}<0.05$, Student's t-test.

of CRC, including cancer cell proliferation, sphere formation, chemoresistance, radioresistance and invasion.

It seems likely that the relevance of ALDOA to cancer proliferation, sphere formation, and radioresistance is based on the function of ALDOA in glycolysis and cell cycle regulation. Other studies have reported that the glycolytic pathway and the expression of glycolytic enzymes are associated with cancer radiosensitivity $(26,27)$. We showed that ALDOA expression is positively correlated with the activity of glycolysis, with cell cycle progression and with ROS production. ALDOA is an isozyme of the aldolase family, and a previous study showed that aldolase is involved in the cell cycle. Its involvement in cell cycle progression is mediated by the binding of aldolase to F-actin, with the cell cycle defects observed at M phase (28). Our flow cytometry data were consistent with these data. As a result of the cell cycle defect at $M$ phase, the G2/G1 ratio increased when ALDOA was knocked down. Both inhibition of the glycolytic pathway and the arrest of cell division caused by ALDOA knockdown induced apoptosis, probably because of the increase in oxidative stress and the inhibition of tumor progression.

As for the relevance of ALDOA in chemoresistance and invasion, we suggested an association of ALDOA expression with EMT progression. Other studies have previously concluded that the EMT correlates with chemoresistance $(29,30)$, thus, it appeared that the association of ALDOA expression with the EMT led to chemoresistance and cancer invasion in CRC cell lines. This seems in line with the observed relationship between ALDOA expression and the clinicopathological features of CRC i.e. the ALDOA high 
A
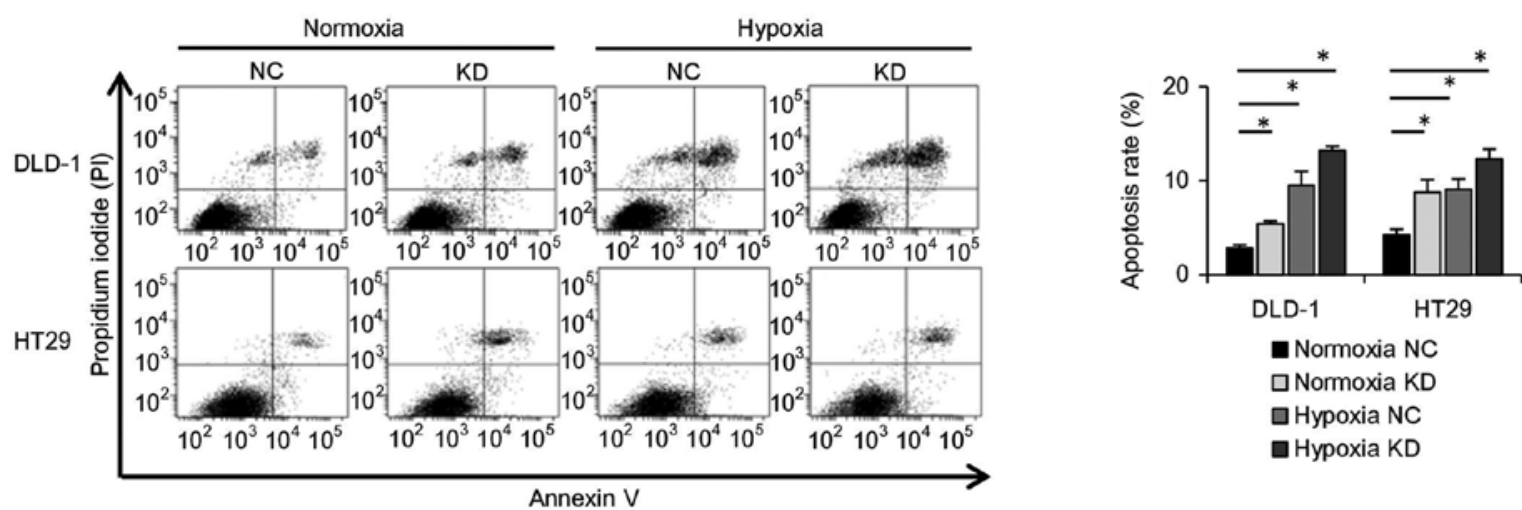

B
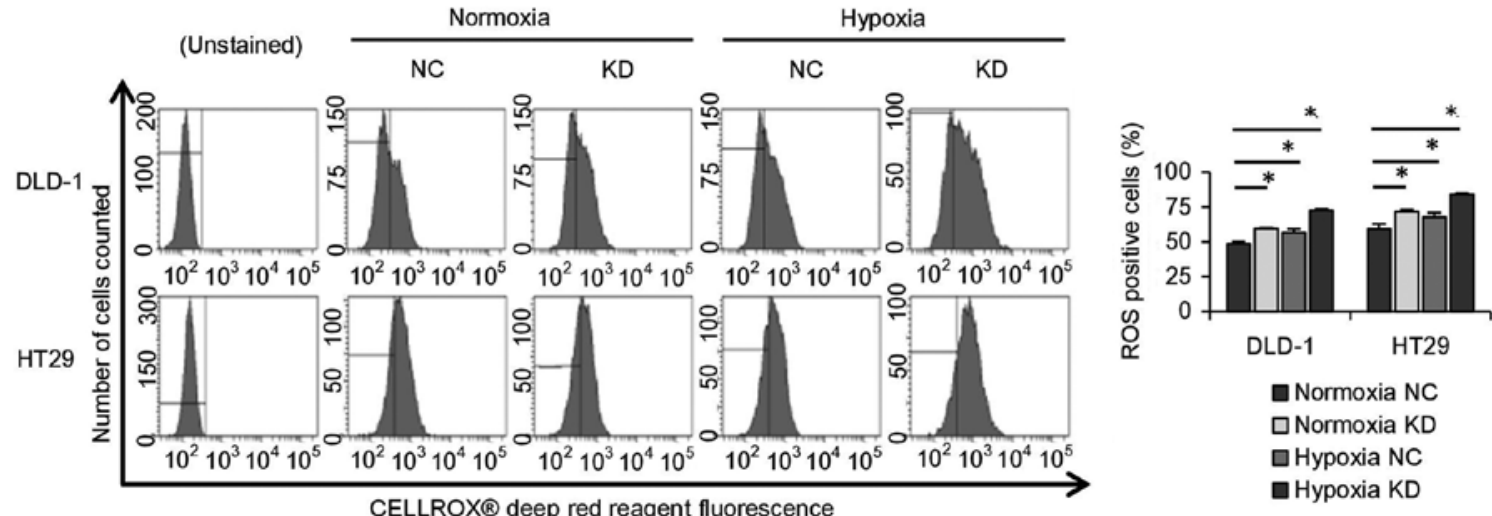

C
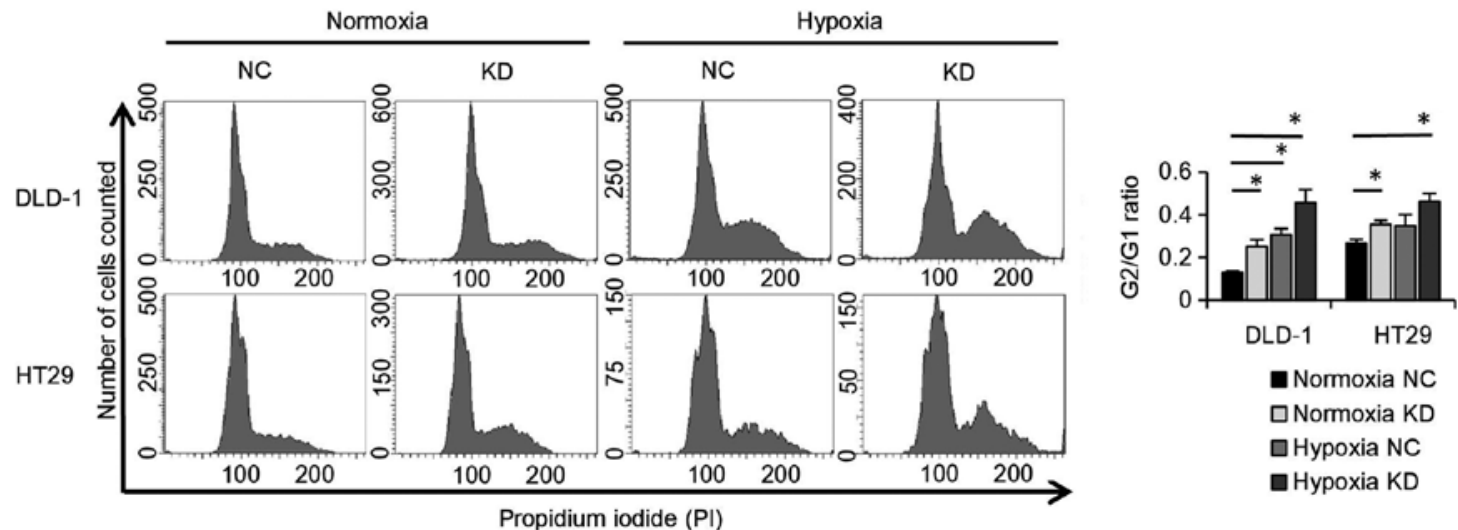

Figure 5. ALDOA expression is associated with cell cycle and apoptosis. (A) Apoptosis in ALDOA knockdown cells in normoxic and hypoxic conditions. (B) Analysis of reactive oxygen species (ROS) activity in ALDOA knockdown cells in normoxic and hypoxic conditions. (C) Cell cycle analysis of ALDOA knockdown cells in normoxic and hypoxic conditions. The results represent the means \pm SD of three independent experiments. KD, ALDOA knockdown; NC, negative control. ${ }^{*} \mathrm{P}<0.05$, Student's t-test.

expression group included more patients with lymph node metastasis than the low expression group (Table I).

ALDOA is a hypoxia-inducible gene and a downstream target of HIF1A. Hypoxia is an important feature in cancer malignancy, and under hypoxic conditions the glycolytic pathway is upregulated by activating HIF1A. Notably, the EMT is upregulated in hypoxic environments (31), while cell cycle progression is inhibited (7); accordingly, the present study verified the effect of ALDOA on cancer under hypoxic conditions. Our data revealed that especially under hypoxic conditions, ALDOA is essential for CRC cell proliferation and sphere formation. On the other hand, knockdown of ALDOA in normoxic conditions resulted in less prolifera- tion and sphere formation, but the gap with the control was not so clear as in hypoxia. It is speculated that in hypoxic conditions, cancer cells may try to adapt to hypoxia by upregulating hypoxia-inducible genes; however, when ALDOA is knocked down, they are unable to adapt, and the growth of cancer cells stops. This implies that particularly in hypoxic conditions, cancer cells could not grow well without ALDOA expression.

In summary, we found that ALDOA positively regulated the activity of the glycolytic pathway and the EMT and was necessary for cell cycle progression. Thus, ALDOA is implicated in CRC proliferation, sphere formation, therapeutic resistance and invasion. 


\section{Acknowledgements}

The present study was supported by the JSPS KAKENHI Grand Number 15K10140 and the Takeda Science Foundation.

\section{References}

1. Weitz J, Koch M, Debus J, Höhler T, Galle PR and Büchler MW: Colorectal cancer. Lancet 365: 153-165, 2005.

2. Beppu N, Yoshie H, Kimura F, Aihara T, Doi H, Kamikonya N, Matsubara N, Tomita N, Yanagi $\mathrm{H}$ and Yamanaka N: Clinicopathological outcomes of preoperative chemoradiotherapy using S-1 plus Irinotecan for T4 lower rectal cancer. Surg Today 46: 852-859, 2016.

3. Saeki H, Emi Y, Kumashiro R, Otsu H, Kawano H, Ando K, Ida S, Kimura Y, Tokunaga E, Oki E, et al: Impact of second-line and later cetuximab-containing therapy and KRAS genotypes in patients with metastatic colorectal cancer: A multicenter study in Japan. Surg Today 44: 1457-1464, 2014.

4. Jemal A, Siegel R, Xu J and Ward E: Cancer statistics, 2010. CA Cancer J Clin 60: 277-300, 2010.

5. Jensen RL: Brain tumor hypoxia: Tumorigenesis, angiogenesis, imaging, pseudoprogression, and as a therapeutic target. J Neurooncol 92: 317-335, 2009.

6. Vaupel P, Briest S and Höckel M: Hypoxia in breast cancer: Pathogenesis, characterization and biological/therapeutic implications. Wien Med Wochenschr 152: 334-342, 2002.

7. Zhang L, Huang G, Li X, Zhang Y, Jiang Y, Shen J, Liu J, Wang Q, Zhu J, Feng X, et al: Hypoxia induces epithelial-mesenchymal transition via activation of SNAI1 by hypoxia-inducible factor $-1 \alpha$ in hepatocellular carcinoma. BMC Cancer 13: 1-9, 2013.

8. Lee GW, Go SI, Cho YJ, Jeong YY, Kim HC, Duk Lee J, Hwang YS, Ko GH, Lee JH, Kim DC, et al: Hypoxia-inducible factor- $1 \alpha$ and excision repair cross-complementing 1 in patients with small cell lung cancer who received front-line platinumbased chemotherapy: A retrospective study. J Thorac Oncol 7: 528-534, 2012.

9. Liang D, Ma Y, Liu J, Trope CG, Holm R, Nesland JM and Suo Z: The hypoxic microenvironment upgrades stem-like properties of ovarian cancer cells. BMC Cancer 12: 201, 2012.

10. Higgins LH, Withers HG, Garbens A, Love HD, Magnoni L, Hayward SW and Moyes CD: Hypoxia and the metabolic phenotype of prostate cancer cells. Biochim Biophys Acta 1787: 1433-1443, 2009.

11. Chang LH, Chen CH, Huang DY, Pai HC, Pan SL and Teng CM: Thrombin induces expression of twist and cell motility via the hypoxia-inducible factor-1 $\alpha$ translational pathway in colorectal cancer cells. J Cell Physiol 226: 1060-1068, 2011.

12. Uemura M, Yamamoto H, Takemasa I, Mimori K, Hemmi H, Mizushima T, Ikeda M, Sekimoto M, Matsuura N, Doki Y, et al: Jumonji domain containing $1 \mathrm{~A}$ is a novel prognostic marker for colorectal cancer: In vivo identification from hypoxic tumor cells. Clin Cancer Res 16: 4636-4646, 2010.

13. Uemura M, Yamamoto H, Takemasa I, Mimori K, Mizushima T, Ikeda M, Sekimoto M, Doki Y and Mori M: Hypoxia-inducible adrenomedullin in colorectal cancer. Anticancer Res 31: 507-514, 2011.

14. Yamamoto H, Tei M, Uemura M, Takemasa I, Uemura $Y$, Murata K, Fukunaga M, Ohue M, Ohnishi T, Ikeda K, et al: Ephrin-A1 mRNA is associated with poor prognosis of colorectal cancer. Int J Oncol 42: 549-555, 2013.
15. Noda T, Yamamoto H, Takemasa I, Yamada D, Uemura M, Wada H, Kobayashi S, Marubashi S, Eguchi H, Tanemura M, et al: PLOD2 induced under hypoxia is a novel prognostic factor for hepatocellular carcinoma after curative resection. Liver Int 32: 110-118, 2012.

16. Munakata K, Uemura M, Takemasa I, Ozaki M, Konno M, Nishimura J, Hata T, Mizushima T, Haraguchi N, Noura S, et al: SCGB2A1 is a novel prognostic marker for colorectal cancer associated with chemoresistance and radioresistance. Int J Oncol 44: 1521-1528, 2014.

17. Tochio T, Tanaka H, Nakata S and Hosoya H: Fructose-1,6bisphosphate aldolase A is involved in HaCaT cell migration by inducing lamellipodia formation. J Dermatol Sci 58: 123-129, 2010.

18. Semenza GL, Roth PH, Fang HM and Wang GL: Transcriptional regulation of genes encoding glycolytic enzymes by hypoxiainducible factor 1. J Biol Chem 269: 23757-23763, 1994.

19. Sharp FR and Bernaudin M: HIF1 and oxygen sensing in the brain. Nat Rev Neurosci 5: 437-448, 2004.

20. Chen X, Yang TT, Zhou Y, Wang W, Qiu XC, Gao J, Li CX, Long H, Ma BA, Ma Q, et al: Proteomic profiling of osteosarcoma cells identifies ALDOA and SULT1A3 as negative survival markers of human osteosarcoma. Mol Carcinog 53: 138-144, 2014.

21. Long F, Cai X, Luo W, Chen L and Li K: Role of aldolase A in osteosarcoma progression and metastasis: In vitro and in vivo evidence. Oncol Rep 32: 2031-2037, 2014.

22. Du S, Guan Z, Hao L, Song Y, Wang L, Gong L, Liu L, Qi $\mathrm{X}$, Hou Z and Shao S: Fructose-bisphosphate aldolase A is a potential metastasis-associated marker of lung squamous cell carcinoma and promotes lung cell tumorigenesis and migration. PLoS One 9: e85804, 2014.

23. Shimizu T, Inoue $K$, Hachiya $H$, Shibuya N, Shimoda $M$ and Kubota K: Frequent alteration of the protein synthesis of enzymes for glucose metabolism in hepatocellular carcinomas. J Gastroenterol 49: 1324-1332, 2014.

24. Munakata K, Uemura M, Tanaka S, Kawai K, Kitahara T, Miyo M, Kano Y, Nishikawa S, Fukusumi T, Takahashi Y, et al: Cancer stem-like properties in colorectal cancer cells with low proteasome activity. Clin Cancer Res 22: 5277-5286, 2016.

25. Warburg O: On the origin of cancer cells. Science 123: 309-314, 1956.

26. Meng MB, Wang HH, Guo WH, Wu ZQ, Zeng XL, Zaorsky NG, Shi HS, Qian D, Niu ZM, Jiang B, et al: Targeting pyruvate kinase M2 contributes to radiosensitivity of non-small cell lung cancer cells in vitro and in vivo. Cancer Lett 356: 985-993, 2015.

27. Pitroda SP, Wakim BT, Sood RF, Beveridge MG, Beckett MA, MacDermed DM, Weichselbaum RR and Khodarev NN: STAT1dependent expression of energy metabolic pathways links tumour growth and radioresistance to the Warburg effect. BMC Med 7: $68,2009$.

28. Ritterson Lew C and Tolan DR: Targeting of several glycolytic enzymes using RNA interference reveals aldolase affects cancer cell proliferation through a non-glycolytic mechanism. J Biol Chem 287: 42554-42563, 2012

29. Fischer KR, Durrans A, Lee S, Sheng J, Li F, Wong ST, Choi H, El Rayes T, Ryu S, Troeger J, et al: Epithelial-to-mesenchymal transition is not required for lung metastasis but contributes to chemoresistance. Nature 527: 472-476, 2015.

30. Singh A and Settleman J: EMT, cancer stem cells and drug resistance: An emerging axis of evil in the war on cancer. Oncogene 29: 4741-4751, 2010

31. Majmundar AJ, Wong WJ and Simon MC: Hypoxia-inducible factors and the response to hypoxic stress. Mol Cell 40: 294-309, 2010. 